Revista de

CIENCIAS AMBIENTALES

Tropical Journal of Environmental Sciences

\title{
Ensambles arbóreos en sistemas agroforestales cafetaleros con diferente intensidad de manejo en Veracruz, México
}

\author{
Tree Assemblages in Agroforestry Systems with Different Intensity of \\ Management in Veracruz, Mexico
}

\author{
Mario J. Gómez-Martínez ${ }^{a}$, Gabriel Díaz-Padilla ${ }^{b}$,Fabien Charbonnierc, \\ Gabriela Sánchez-Viveros ${ }^{d}$, Carlos Roberto Cerdán-Cabrera ${ }^{e}$
}

a Estudiante del Doctorado en Ciencias Agropecuarias, Facultad de Ciencias Agrícolas, Universidad Veracruzana, México. Profesor Asistente, Laboratorio de Ecoagricultura, Grupo de Investigación en Sistemas Agroforestales Pecuarios, Departamento de Producción y Sanidad Vegetal, Facultad de Ingeniería Agronómica, Universidad del Tolima, Colombia. ORCID: 0000-0002-5048-7182. mjgomez@ut.edu.co

b Investigador, Instituto Nacional de Investigaciones Forestales Agrícolas y Pecuarias (INIFAP), Veracruz, México. diaz.gabriel@inifap.gob.mx Académico e investigador, Cátedra CONACYT, El Colegio de la Frontera Sur (ECOSUR), Chiapas, México. charbonnier@ecosur.mx Académica, Facultad de Ciencias Agrícolas, Universidad Veracruzana, México. gabsanchez@uv.mx

Académico, Facultad de Ciencias Agrícolas, Universidad Veracruzana, México. ORCID: 0000-0002-0498-2617. ccerdan@uv.mx

Director y Editor:

Dr. Sergio A. Molina-Murillo

Consejo Editorial:

Dra. Mónica Araya, Costa Rica Limpia, Costa Rica Dr. Gerardo Ávalos-Rodríguez. SFS y UCR, USA y Costa Rica Dr. Olman Murillo Gamboa, ITCR, Costa Rica

Dr. Luko Hilje, CATIE, Costa Rica

Dr. Arturo Sánchez Azofeifa. Universidad de Alberta-Canadá

Asistente:

Joseline Jimenez Brenes

Editorial:

Editorial de la Universidad Nacional de Costa Rica (EUNA)

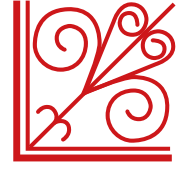

euna

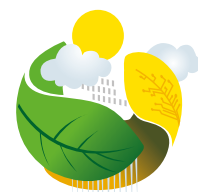

A

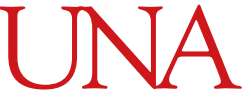

UNIVERSIDAD

NACIONAL

C O S T A R I C A 


\title{
Ensambles arbóreos en sistemas agroforestales cafetaleros con diferente intensidad de manejo en Veracruz, México
}

\author{
Tree Assemblages in Agroforestry Systems with Different Intensity of Management \\ in Veracruz, Mexico \\ Mario J. Gómez-Martínez ${ }^{a}$, Gabriel Díaz-Padilla ${ }^{b}$,Fabien Charbonnierc, \\ Gabriela Sánchez-Viveros ${ }^{d}$, Carlos Roberto Cerdán-Cabrera ${ }^{e}$
}

[Recibido: 28 de setiembre, 2017. Aceptado: 4 de diciembre, 2017. Corregido: 1 de febrero, 2018. Publicado: 1 de julio, 2018.]

Resumen

El potencial de los sistemas agroforestales (SAF) en la conservación de la diversidad biológica es ampliamente reconocido. Existen abundantes reportes sobre cómo la complejidad en la cobertura forestal de los SAF se relaciona con la abundancia de diferentes taxa. Sin embargo, hay poca información del vínculo entre esta complejidad con la intensificación del manejo. Se evaluaron la riqueza y abundancia de los árboles utilizados como sombra en 15 parcelas de 20 x 50 m en SAF, con el objetivo de entender las variaciones del ensamblaje arbóreo en fincas con manejo heterogéneo, en una zona originalmente de bosque de niebla, con aproximadamente 200 años de haberse introducido el café. Se tomaron muestras botánicas de todos los árboles con un diámetro de $\geq 10 \mathrm{~cm}$ a la altura del pecho. En total se registraron 178 individuos arbóreos, incluidos en 17 familias, 24 géneros y 33 especies. Se destaca la familia Fabaceae que con 11 especies reunió el $60 \%$ de la abundancia. El género más común por su riqueza es Inga, con 6 especies. El ensamblaje de las especies arbóreas en los SAF cafetaleros es influenciado por las decisiones de los productores respecto a la productividad de sus parcelas, quienes principalmente dirigen sus esfuerzos a la productividad de los cafetos, manteniendo la cobertura arbórea, lo que es positivo para la conservación de biodiversidad y provisión de servicios ecosistémicos.

Palabras clave: abundancia; Coffea arabica; diversidad arbórea; género Inga; riqueza.

Abstract

The potential of agroforestry systems (AFS) to conserve biological diversity is widely recognized. There are abundant reports about how tree cover complexity within AFS is related to the abundance of several taxa, but there is scant information regarding how this complexity is related to management intensification. Shade-tree abundance and richness of 15 plots $(20 \times 50 \mathrm{~m})$ were surveyed with the aim to understand how tree assemblage changes in farms under different management conditions, within a cloud forest formerly zone, which changed to coffee plantations approximately 200 years ago. There was a total of 178 tree individuals, included in 17 families, 24 genera and 33 species. Fabaceae family overcame with 11 species, reaching $60 \%$ of the total abundance. Inga was the most common genus with six species. Tree species assemblage within coffee-ASF is highly related to farmers' decisions

\footnotetext{
a Estudiante del Doctorado en Ciencias Agropecuarias, Facultad de Ciencias Agrícolas, Universidad Veracruzana, México. Profesor Asistente, Laboratorio de Ecoagricultura, Grupo de Investigación en Sistemas Agroforestales Pecuarios, Departamento de Producción y Sanidad Vegetal, Facultad de Ingeniería Agronómica, Universidad del Tolima, Colombia. ORCID: 0000-0002-5048-7182. mjgomez@ut.edu.co b Investigador, Instituto Nacional de Investigaciones Forestales Agrícolas y Pecuarias (INIFAP), Veracruz, México. diaz.gabriel@inifap.gob.mx c Académico e investigador, Cátedra CONACYT, El Colegio de la Frontera Sur (ECOSUR), Chiapas, México. charbonnier@ecosur.mx

d Académica, Facultad de Ciencias Agrícolas, Universidad Veracruzana, México. gabsanchez@uv.mx

e Académico, Facultad de Ciencias Agrícolas, Universidad Veracruzana, México. ORCID: 0000-0002-0498-2617. ccerdan@uv.mx
}

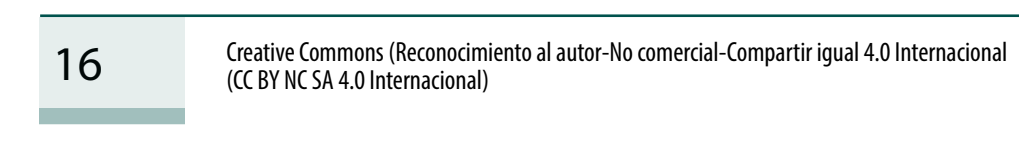


regarding productivity, whilst tree cover is kept. This is a positive fact in order to conserve biodiversity and supply ecosystem services.

Keywords: abundance; Coffea arabica; Inga genus; tree diversity; richness.

\section{Introducción}

La intensificación agrícola es un conjunto de patrones de cambio en el uso del suelo con la característica de un mayor empleo de los mismos recursos para la producción agrícola, generalmente como resultado de una modificación de cultivo intermitente a continuo en la misma área de tierra. La intensificación se da mediante el uso de variedades de cultivos de alta productividad, fertilizantes y pesticidas químicos, irrigación y mecanización, generando el aumento de los rendimientos tanto en los países desarrollados como en los menos desarrollados. Sin embargo, han surgido preocupaciones sobre la sostenibilidad a largo plazo y las consecuencias ambientales de la intensificación de los sistemas agrícolas (Matson, Parton, Power y Swift, 1997). El cultivo del café, uno de los sistemas agrícolas de gran importancia económica, social y ambiental en Latinoamérica, no es la excepción en este proceso. Para contrarrestar la disminución de la productividad en la caficultura, los institutos técnicos de varios países han recomendado una serie de medidas de intensificación de las plantaciones: reemplazo de variedades tradicionales por cultivares productivos y resistentes a la roya, paquetes de fertilizantes y pesticidas, así como la remoción de la sombra (López et al., 2013a).

Tradicionalmente, la caficultura se desarrolla bajo la sombra de árboles, en ocasiones debajo de doseles en bosques, ya que el café en su hábitat original (en los altiplanos de Etiopía) es una planta de sotobosque. Sin embargo, experiencias en plantaciones de café a pleno sol resultaron bastante exitosas en Brasil o Colombia. La disminución de la sombra es, entonces, una solución promovida en otros países, como Perú, México y El Salvador, para aumentar la productividad y reducir el impacto de enfermedades generalmente asociadas con la sombra (roya anaranjada del cafeto); no obstante, hay una falta de consenso sobre este tema (Avelino et al., 2015; Charbonnier et al., 2017), ya que el costo de este proceso de intensificación de los cafetales es una reducción de la resiliencia del agroecosistema, mientras que, paradójicamente, el cambio climático (CC) requiere una mayor capacidad de respuesta de los cafetos, para evitar la disminución del rendimiento de las plantaciones (Altieri, Nicholls, Henao y Lana, 2015).

Las estrategias de adaptación al CC recomiendan el mantenimiento y la promoción de los árboles de sombra en la agricultura, ya que estos favorecen la redundancia ecológica y proveen servicios de regulación del microclima (Tscharntke et al., 2011). El ensamble arbóreo, es decir, todos los árboles de sombra que se encuentran en las plantaciones de café, es crucial para la sostenibilidad y resiliencia del sistema agroforestal. Dependiendo del ensamble arbóreo, las plantaciones de café pueden prestar servicios ecosistémicos tanto de regulación como de provisión y conservar la biodiversidad asociada. En efecto, diversos estudios han evidenciado que el componente arbóreo presente en las plantaciones de café podría brindar condiciones microclimáticas más clementes y más favorables para la resiliencia del sistema agroforestal cafetalero (García-Mayoral, Valdez-Hernández, Luna-Cavazos y López-Morgado, 2015; López-Gómez, 
Williams-Linera y Manson, 2008; Méndez, Gliessman y Gilbert, 2007; Perfecto y Vandermeer, 2015; Romero-Alvarado, Soto-Pinto, García-Barrios y Barrera-Gaitán, 2002; Siles, Harmand y Vaast, 2010).

Actualmente, la caficultura necesita definir un proceso de intensificación ecológica, que pueda a la vez mantener una productividad de café alta, sin impactar en la función de los cafetales como proveedores de hábitat para diversos grupos taxonómicos, maximizar la generación de servicios ambientales (Marin-Castro, Geissert, Negrete-Yankelevich y Chávez, 2016; Perfecto y Vandermeer, 2015) y, finalmente, adaptarse a las condiciones climáticas cambiantes. En este sentido, Philpott et al. (2008) recomiendan que se proteja el componente arbóreo de los SAF, se promueva la producción de café bajo sombras tradicionales (llamadas rústicos) y se restauren las fincas intensivas, mediante el incremento de la densidad y riqueza de árboles nativos (fortalecer el ensamble arbóreo), puesto que se ha reportado que el café de alta biodiversidad es también de alta rentabilidad (Gordon, Manson, Sundberg y Cruz-Angón, 2007).

En México, existe en los agroecosistemas de café una tremenda variación en la estructura y diversidad del estrato arbóreo y arbustivo, lo cual es una oportunidad formidable para estudiar la disyuntiva entre rendimiento del café y maximización de los servicios ecosistémicos de apoyo y de regulación. En consecuencia, dado el potencial crucial de los sistemas agroforestales (SAF) en la adaptación al CC, existe la urgencia de recopilar y sistematizar informaciones climáticas, botánicas, estructurales y los servicios ecosistémicos que proveen en asociación con el café, para producir recomendaciones más útiles y pertinentes dirigidas al diseño y rediseño de SAF cafetaleros. El vacío de información es urgente suplirlo; para ello, se debe aumentar la investigación en las regiones productoras de café donde predominan los SAF y, con ello, aumentar los rendimientos de las plantaciones y disminuir la pérdida de estos agroecosistemas. Es por lo anterior que, en la región cafetalera de Coatepec, una de las zonas cafetaleras más importantes de México, se desarrolló esta investigación, con el objetivo de proporcionar información de la riqueza y abundancia de las especies arbóreas, así como de mostrar el ensamble de árboles en fincas con variado manejo de las plantaciones en los SAF de café. Esto último con el afán de tener información útil para el diseño de SAF cafetaleros que cumplan, simultáneamente, con sus funciones de conservación y de producción.

\section{Metodología}

\section{1 Área de estudio}

El estudio se localizó en los municipios de Coatepec, Xico, Teocelo y Emiliano Zapata, los cuales concentran la mayor superficie con café, dentro de la región cafetalera de Coatepec, Veracruz, México. En esta zona, se cultiva, principalmente, Coffea arabica y cuenta con cerca de 12000 cafeticultores (López et al., 2013a). La zona de estudio se encuentra en bosque mesófilo de montaña, con temperatura promedio anual de 16 a $23^{\circ} \mathrm{C}$ y rango de precipitación promedio anual de entre 1330 y $2107 \mathrm{~mm}$, con de 2 a 4 meses lluviosos por año (de junio a septiembre). El café fue introducido en esta área hace aproximadamente 200 años, lo cual

\begin{tabular}{|c|c|c|}
\hline 18 & $\begin{array}{l}\text { Creative Commons (Reconocimiento al autor-No comercial-Compartir igual } 4.0 \text { Internacional } \\
\text { (CC BY NC SA } 4.0 \text { Internacional) }\end{array}$ & $\begin{array}{l}\text { Revista de } \\
\text { CIENCIAS AMBIENTALES } \\
\text { Tropical Journal of Environmental Sciences }\end{array}$ \\
\hline
\end{tabular}


hace que: a) los productores estén familiarizados con el cultivo, y b) la cobertura arbórea original está muy modificada.

En el área de estudio se seleccionaron 15 parcelas de 0,1 ha (50 x $20 \mathrm{~m})$, en cada una se marcaron 3 transectos en banda de $50 \mathrm{~m} \mathrm{x} 2 \mathrm{~m}$ (en total $300 \mathrm{~m}^{2}$ ), dos ubicados en los extremos y uno en el centro de ella, separados a $7 \mathrm{~m}$. Todas las parcelas se encuentran dentro de fincas de productores de café y son representativas de los diversos manejos. Previa a la selección de las parcelas y debido a la ausencia de cafetales a pleno sol, se hizo una clasificación a priori de los SAF cafetaleros más representativos de la región. De acuerdo con un gradiente de manejo y complejidad arbórea, se conformaron 3 tipos de SAF, los cuales se clasificaron como 1) tradicional, 2) comercial e 3) intensivo (Cuadro 1). Estos 3 tipos de SAF se asemejan a los llamados cafetal rústico, policultivo diverso y policultivo simple, descritos por Escamilla et al. (1995) y López et al. (2013a). La intensidad de manejo en cada uno de los tipos de SAF se consideró según el número de cafetos, variedades, uso de fertilizantes y podas, siendo menor en los SAF tradicionales y mayor en los intensivos. Caso contrario resultó para la complejidad arbórea, que pasó de mayor a menor densidad de individuos, riqueza florística, altura de los árboles y densidad de las copas del manejo tradicional al intensivo. El manejo tradicional reunió a las estructuras del cafetal rústico y policultivo diverso; el comercial se conformó con la estructura policultivo simple, y el intensivo estuvo dado por la estructura de monocultivo de café bajo sombra. Aunque el número de parcelas no se seleccionó proporcional a la abundancia de cada tipo de SAF en la región, se muestrearon 5 parcelas por cada uno de los 3 tipos de sistemas. 
Revista de Ciencias Ambientales (Trop J Environ Sci). (Julio-Diciemnbre, 2018). EISSN: 2215-3896. Vol 52(2): 16-38.

DOl: http://dx.doi.org/10.15359/rca.52-2.2

URL: www.revistas.una.ac.cr/ambientales

EMAIL: revista.ambientales@una.cr

Cuadro 1. Características de los 3 tipos de sistemas agroforestales cafetaleros en Veracruz, México

\begin{tabular}{|c|c|c|c|c|c|c|c|}
\hline \multirow{3}{*}{ Variable } & \multirow{3}{*}{ Unidad } & \multicolumn{6}{|c|}{ Gradiente de manejo de sistemas agroforestales } \\
\hline & & \multicolumn{2}{|c|}{ Tradicional } & \multicolumn{2}{|c|}{ Comercial } & \multicolumn{2}{|c|}{ Intensivo } \\
\hline & & Media & DE & Media & DE & Media & $\mathrm{DE}$ \\
\hline Densidad & \# de cafetos ha ${ }^{-1}$ & 3820 & 1744.0 & 3847 & 1556.1 & 7433 & 945.2 \\
\hline Edad de cafetos & años & 18 & 11.6 & 13 & 7.2 & 13 & 2.1 \\
\hline $\mathrm{N}$ & $\%$ & 0.7 & 0.1 & 0.3 & 0.2 & 0,2 & 0.1 \\
\hline $\mathrm{P}$ & $\mathrm{mg} \mathrm{kg}^{-1}$ & 9.0 & 6.2 & 26.0 & 32.4 & 68.8 & 17.8 \\
\hline $\mathrm{K}$ & $\mathrm{mg} \mathrm{kg}^{-1}$ & 194.7 & 16.0 & 211.9 & 76.5 & 567.0 & 674.1 \\
\hline $\mathrm{Ca}$ & $\mathrm{mg} \mathrm{kg}^{-1}$ & 1134.2 & 402.5 & 1149.4 & 671.0 & 629.2 & 310.2 \\
\hline $\mathrm{Mg}$ & $\mathrm{mg} \mathrm{kg}^{-1}$ & 916.4 & 161.1 & 849.3 & 207.8 & 575.8 & 112.8 \\
\hline $\mathrm{Zn}$ & $\mathrm{mg} \mathrm{kg}^{-1}$ & 2.5 & 0.5 & 2.3 & 1.4 & 2.9 & 0.5 \\
\hline $\mathrm{Al}$ & $\mathrm{mg} \mathrm{kg}^{-1}$ & 145.7 & 67.7 & 124.1 & 56.0 & 102.5 & 48.3 \\
\hline MO & $\%$ & 4.0 & 1.4 & 3.4 & 1.4 & 2.7 & 0.5 \\
\hline $\mathrm{CO}$ & $\%$ & 6.8 & 2.4 & 5.8 & 2.4 & 4.6 & 0.8 \\
\hline Altitud & m s.n.m. & 1180.6 & 71.4 & 1131.6 & 151.0 & 1151.4 & 10.3 \\
\hline Localidad & & \multicolumn{2}{|c|}{ Coatepec y Xico } & \multicolumn{2}{|c|}{ Coatepec y Teocelo } & \multicolumn{2}{|c|}{ Emiliano Zapata } \\
\hline Variedades de café & $\%$ & \multicolumn{2}{|c|}{$\begin{array}{l}\text { Mundo Novo (25), } \\
\text { Arábica (23), Catimor } \\
\text { (21), Costa Rica } 95 \text { (19), } \\
\text { Caturra (10), Sarchimor } \\
\text { (1) y Garnica (1) }\end{array}$} & \multicolumn{2}{|c|}{$\begin{array}{l}\text { Sarchimor (43), Costa } \\
\text { Rica } 95 \text { (37), Catimor (9), } \\
\text { Caturra (9), Mondo Novo } \\
\text { (1) y Oro Azteca (1) }\end{array}$} & \multicolumn{2}{|c|}{$\begin{array}{c}\text { Catimor (46), Sarchimor } \\
\text { (40), Costa Rica 95 (13) y } \\
\text { Caturra (1) }\end{array}$} \\
\hline Fertilización & $\begin{array}{l}\text { kg N hectárea } \\
\mathrm{año}^{-1}\end{array}$ & 152.9 & 115.2 & 185.1 & 87.1 & 237.9 & 30.2 \\
\hline Control de arvenses & & \multicolumn{2}{|c|}{$\begin{array}{c}\text { Herbicida o chapeo } 1 \text { vez } \\
\text { al año }\end{array}$} & \multicolumn{2}{|c|}{$\begin{array}{l}\text { Herbicida o chapeo } 1 \text { vez } \\
\text { al año }\end{array}$} & \multicolumn{2}{|c|}{ Herbicida 3 veces al año } \\
\hline Poda de cafetos & & \multicolumn{2}{|c|}{ Ninguna } & \multicolumn{2}{|c|}{$\begin{array}{c}\text { Poda de descope o poda } \\
\text { esquelética cada } 4 \text { u } 8 \\
\text { años }\end{array}$} & \multicolumn{2}{|c|}{$\begin{array}{l}\text { Poda esquelética cada } 4 \\
\text { años }\end{array}$} \\
\hline
\end{tabular}

\subsection{Determinación botánica de las especies arbóreas}

Se identificaron con etiquetas y se geolocalizaron todos los individuos arbóreos con un diámetro a la altura del pecho (DAP), superior a $10 \mathrm{~cm}$, que se encontraban dentro de los transectos en banda. Para la determinación botánica de los árboles, se tomaron muestras de las hojas, las flores y los frutos para ser comparadas con especímenes representativos de herbario recogidos y depositados en los herbarios de la Universidad Veracruzana y del Instituto de Ecología en Xalapa, Veracruz. La taxonomía de los árboles fue según el Angiosperm Phylogeny Group IV (APG IV, 2016) para las familias y las especies, según Pennington y Sarukhán (2005) y Tropicos.org (Tropicos.org, 2017). Para el género Inga se utilizaron las claves de Sousa (2009).

Las especies arbóreas se clasificaron como nativas y no nativas. Las especies nativas se encuentran naturalmente en bosques tropicales de montano bajo o premontano de Veracruz, mientras que las no nativas son procedentes de cualquier otro tipo de vegetación o región (López-Gómez et al., 2008). Las especies arbóreas se agruparon también según el síndrome de dispersión de las 
semillas como barocoría (semillas dispersadas por gravedad), zoocoría (semillas dispersadas por los animales) o anemocoría (semillas dispersadas por el viento). La categoría de riesgo de las especies arbóreas se corroboró con la Norma Oficial Mexicana NOM-059-SEMARNAT-2010 de la Secretaría del Medio Ambiente y Recursos Naturales de México.

\subsection{Análisis estadístico}

\subsubsection{Riqueza, abundancia y diversidad}

Se calculó la riqueza, abundancia y diversidad de los árboles para $300 \mathrm{~m}^{2}$ (el área de los 3 transectos en banda por parcela) para homogenizar el esfuerzo de muestreo. Se realizaron curvas de acumulación de especies leñosas, con el propósito de estimar la riqueza y el esfuerzo de muestreo acumulado en cada una de las 15 parcelas, utilizando los estimadores no paramétricos Jacknife 2, Chao 1 y ACE, por medio del programa Estimates versión 9.1.0 (Colwell, 2013). Para plantas leñosas en cafetales y con tamaños de muestra pequeños, Jacknife 2 es el estimador más preciso y menos sesgado (López-Gómez y Williams-Linera, 2006). Los estimadores Chao 1 y ACE se utilizan cuando se obtiene abundancia.

En todos los 5 transectos $\left(1500 \mathrm{~m}^{2}\right)$ de cada tipo de SAF, se estimó la diversidad de especies arbóreas, por medio de curvas de interpolación (rarefacción) sobre la extrapolación (R/E), basadas en los 3 primeros números de Hill (Chao et al., 2014). Este método permite extrapolar la riqueza efectiva de especies a un número determinado de individuos (en este estudio fue de 100), para ver el comportamiento de las curvas (asíntota). Esto se realizó para cada uno de los 3 tipos de SAF, en relación con el número de individuos registrados por parcela y con un intervalo de confianza del $95 \%$, tanto para la riqueza efectiva $\left({ }^{\circ} \mathrm{D}\right)$ como para el exponente de la diversidad de Shannon $\left({ }^{1} \mathrm{D}\right)$, así como para el inverso del índice de Simpson $\left({ }^{2} \mathrm{D}\right)$.

Los números de Hill forman parte de las llamadas diversidades verdaderas ( $\left.{ }^{9} \mathrm{D}\right)$, los cuales permiten calcular la cantidad efectiva de especies de una comunidad (Hill, 1973), y se denominan números equivalentes de los índices de diversidad (Jost, 2006). Chao et al. (2014) propusieron, refiriéndose a los números de Hill, las curvas de R/E basadas en el tamaño de la muestra y la cobertura, como un marco unificado para estimar la diversidad de especies y hacer comparaciones estadísticas acordes con estas estimaciones (Hsieh, Ma y Chao, 2016). Los 3 primeros números de la serie de Hill se obtuvieron con la Ecuación 1, de la siguiente manera:

$$
{ }^{q} D=\left(\sum_{i=1}^{\mathrm{s}} P_{i}^{q}\right)^{\frac{1}{(1-q)}}
$$

Donde ${ }^{q} \mathrm{D}$ es la riqueza efectiva de especies y q es un parámetro que determina la sensibilidad de $\mathrm{D}$ a la abundancia relativa de las especies. La ${ }^{0} \mathrm{D}$ es cuando $\mathrm{q}=0$, que representa una condición en la cual las especies están presentes al menos con un individuo; consecuentemente, solo las especies detectadas contribuyen a la estimación de $\mathrm{D}$. La riqueza efectiva cuando $\mathrm{q}=1$ $\left({ }^{1} \mathrm{D}\right)$ es indefinida, no obstante, el límite de $\mathrm{q}$ tiende a 1 . El estimador ${ }^{1} \mathrm{D}$ es altamente sensible a la frecuencia de especies con abundancia baja o media (Jost, 2006). Para $q=2$, la diversidad $\left({ }^{2} \mathrm{D}\right)$ 
es sensible a la presencia de especies comunes y dominantes (Chao et al., 2014; Jost, 2006). Los análisis de interpolación (rarefacción)/extrapolación se realizaron con el paquete iNEXT (Hsieh et al., 2016) del software R versión 3.3.3 (R Core Team, 2017). Se comparó la densidad, número de familias, riqueza y abundancia de los árboles nativos y su respectivo síndrome de dispersión de semillas por grupo de SAF, mediante un análisis de varianza (ANDEVA) y pruebas de comparación de medias Tukey (LSD, $\alpha=0,05)$. Los análisis estadísticos se realizaron a través del programa InfoStat Versión 2016.

\subsubsection{Composición de las especies}

El grado de recambio de las especies (diversidad beta) entre los tipos de manejo se determinó por medio del índice de Sørensen (similitud) y por el índice de complementariedad (Colwell y Coddington, 1994). El índice de Sørensen compara el número de especies compartidas con respecto a todas las encontradas en los 2 sitios (Jost, Chao y Chazdon, 2011) y se obtuvo por medio de la Ecuación 2 de la siguiente manera:

$$
\text { Is }=2 \mathrm{C} /(\mathrm{A}+\mathrm{B})
$$

Donde A es el número de especies del sitio A, B es el número de especies del sitio B y $\mathrm{C}$ es el número de especies en común entre los sitios A y B.

El índice de complementariedad mide el grado de recambio en la composición de especies entre diferentes hábitats. Este se obtuvo relacionando el número de especies en un área A con el de especies en un área $\mathrm{B}$ y aquel en común entre A y B. De esta forma, la riqueza total para ambos hábitats combinados se calculó con la Ecuación 3, de la siguiente manera:

$$
\mathrm{S}_{\mathrm{AB}}=\mathrm{A}+\mathrm{B}-\mathrm{C}
$$

El número de especies únicas (U) a cualquiera de los dos sitios se calculó, como sigue, con la Ecuación 4:

$$
\mathrm{U}_{\mathrm{AB}}=\mathrm{A}+\mathrm{B}-2 \mathrm{C}
$$

A partir de estos dos valores, se calculó, con la Ecuación 5, la complementariedad de los sitios A y B. Los valores obtenidos en la Ecuación 4 varían desde 0, cuando ambos SAF son idénticos en composición de especies leñosas, hasta 1, cuando las especies arbóreas de los SAF son completamente distintas, expresando qué tanto se complementan los tipos de SAF (Colwell y Coddington, 1994). La complementariedad se calculó de la siguiente manera:

$$
\mathrm{C}_{\mathrm{AB}}=\mathrm{U}_{\mathrm{AB}} / \mathrm{S}_{\mathrm{AB}}
$$

Para comparar la composición, abundancia y uniformidad de especies arbóreas entre los tipos de SAF cafetaleros, se realizaron curvas de rango-abundancia - CRA- (gráficos de Whittaker). Por cada tipo de SAF, se graficaron la abundancia contra el rango de la especie, desde la 
más a la menos abundante. Se seleccionó 1 de los 5 modelos de abundancia (Null, Preemption, Lognormal, Zipf y Mandelbrot) al que ajustaron las CRA por medio del Criterio de Información de Akaike (Akaike Information Criterion [AIC]). El AIC asigna penalidades que corresponden con el exceso de parámetros que utiliza un modelo, algo que no hace el test asintótico de la chi-cuadrado. Es decir, dado un conjunto de modelos construidos con los mismos datos, el AIC los ordena según su verosimilitud basada en los datos con que se construyen (Magurran y McGill, 2011). El modelo que mejor se ajustó y, por lo tanto, el que se seleccionó fue el que tuvo el menor valor de AIC. Las CRA y el ajuste a los modelos se realizó con el paquete Vegan 2.4-3 (Oksanen et al., 2017) en el software R versión 3.3.3 (R Core Team, 2017). En este paquete, los nombres de los modelos corresponden a los citados en la literatura: Null (modelo de vara quebrada - Broken Stick Model), Preemption (serie geométrica = modelo de Motomura = modelo de preferencia de nicho), Lognormal (distribución Lognormal = distribución de Preston), Zipf (ley de Zipf) y Mandelbrot (modelo Zipf-Mandelbrot).

\section{Resultados}

\subsection{Riqueza, abundancia y diversidad}

En los SAF estudiados se registró un total de 178 individuos, representando 17 familias, 24 géneros y 33 especies de árboles y arbustos (Cuadro 2). Se destaca la familia Fabaceae con 11 especies, que reúne el $60 \%$ de la abundancia presente en las parcelas, seguida por Moraceae con 4 especies ( $2 \%$ de la abundancia total). Los géneros con más especies son Inga (6), Ficus (4) y Citrus (2). Las especies más abundantes son Inga vera (37\% de los individuos totales), Lonchocarpus guatemalensis (6\%), Inga jinicuil (6\%), Trema micrantha (6\%) y Heliocarpus appendiculatus (5\%) (Cuadro 2). 
Revista de Ciencias Ambientales (Trop J Environ Sci). (Julio-Diciemnbre, 2018). EISSN: 2215-3896. Vol 52(2): 16-38.

DOI: http://dx.doi.org/10.15359/rca.52-2.2

URL: www.revistas.una.ac.cr/ambientales

EMAIL: revista.ambientales@una.cr

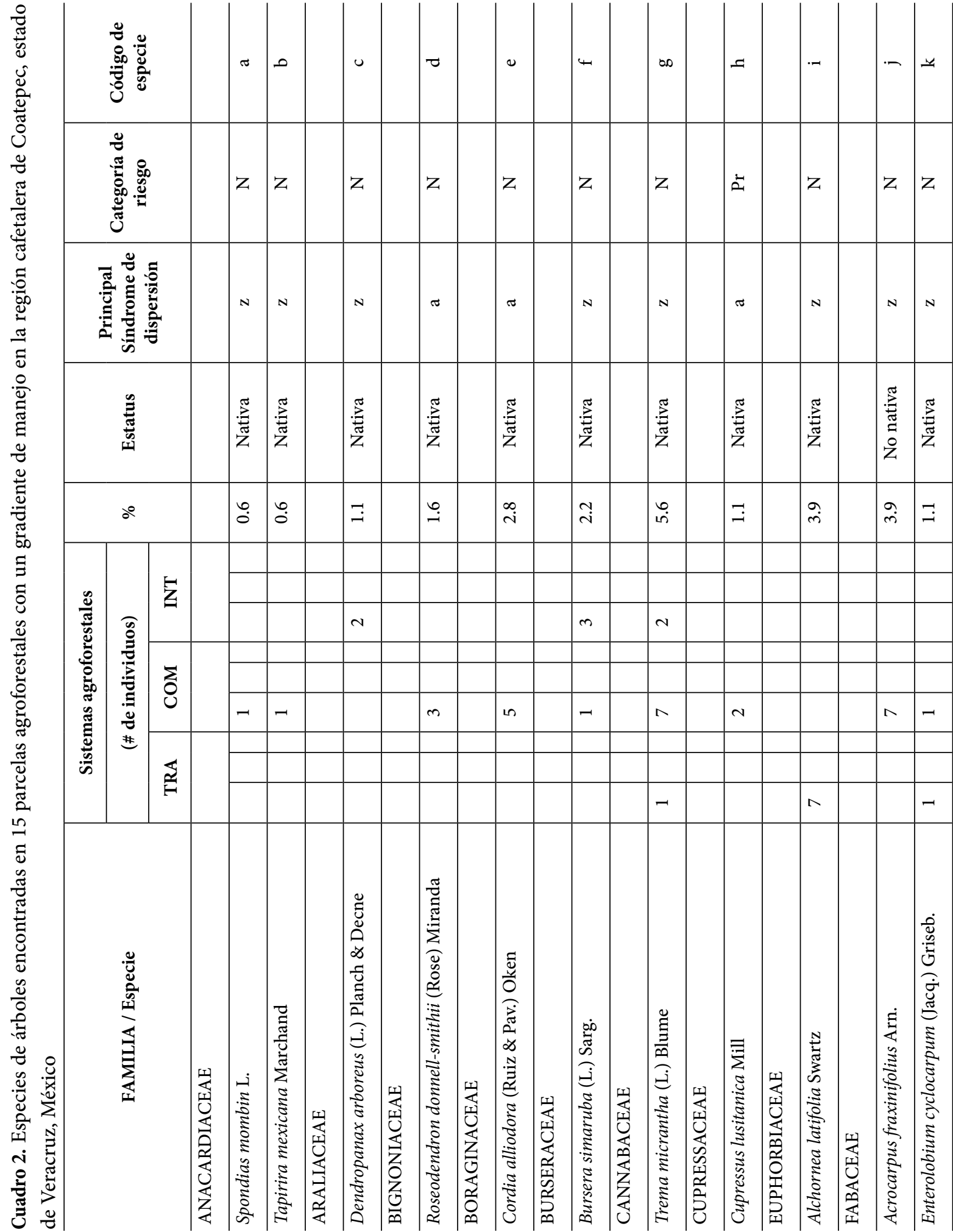


Revista de Ciencias Ambientales (Trop J Environ Sci). (Julio-Diciemnbre, 2018). EISSN: 2215-3896. Vol 52(2): 16-38.

D0l: http://dx.doi.org/10.15359/rca.52-2.2

URL: www.revistas.una.ac.cr/ambientales

EMAlL: revista.ambientales@una.cr

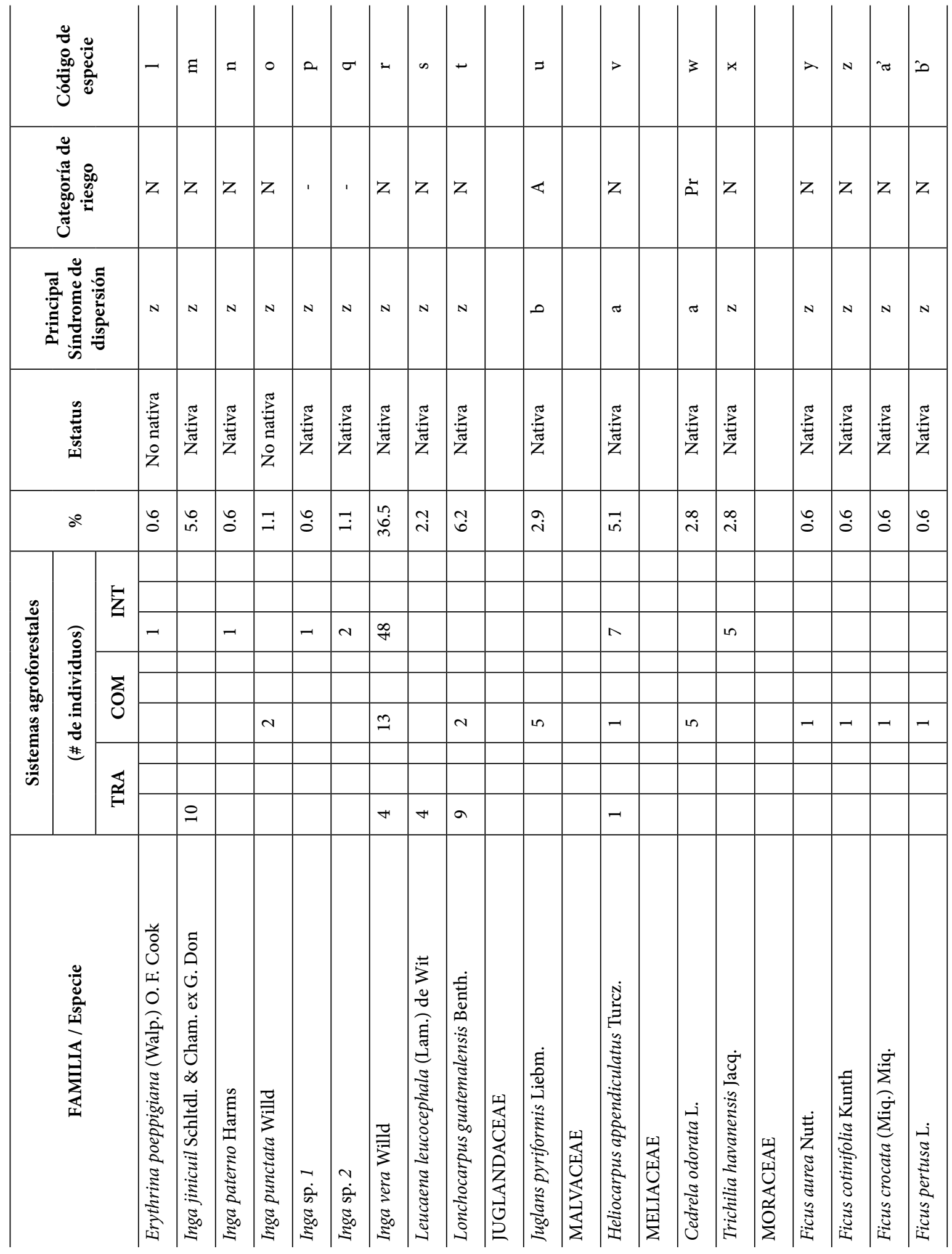


Revista de Ciencias Ambientales (Trop J Environ Sci). (Julio-Diciemnbre, 2018). EISSN: 2215-3896. Vol 52(2): 16-38.

DOI: http://dx.doi.org/10.15359/rca.52-2.2

URL: www.revistas.una.ac.cr/ambientales

EMAIL: revista.ambientales@una.cr

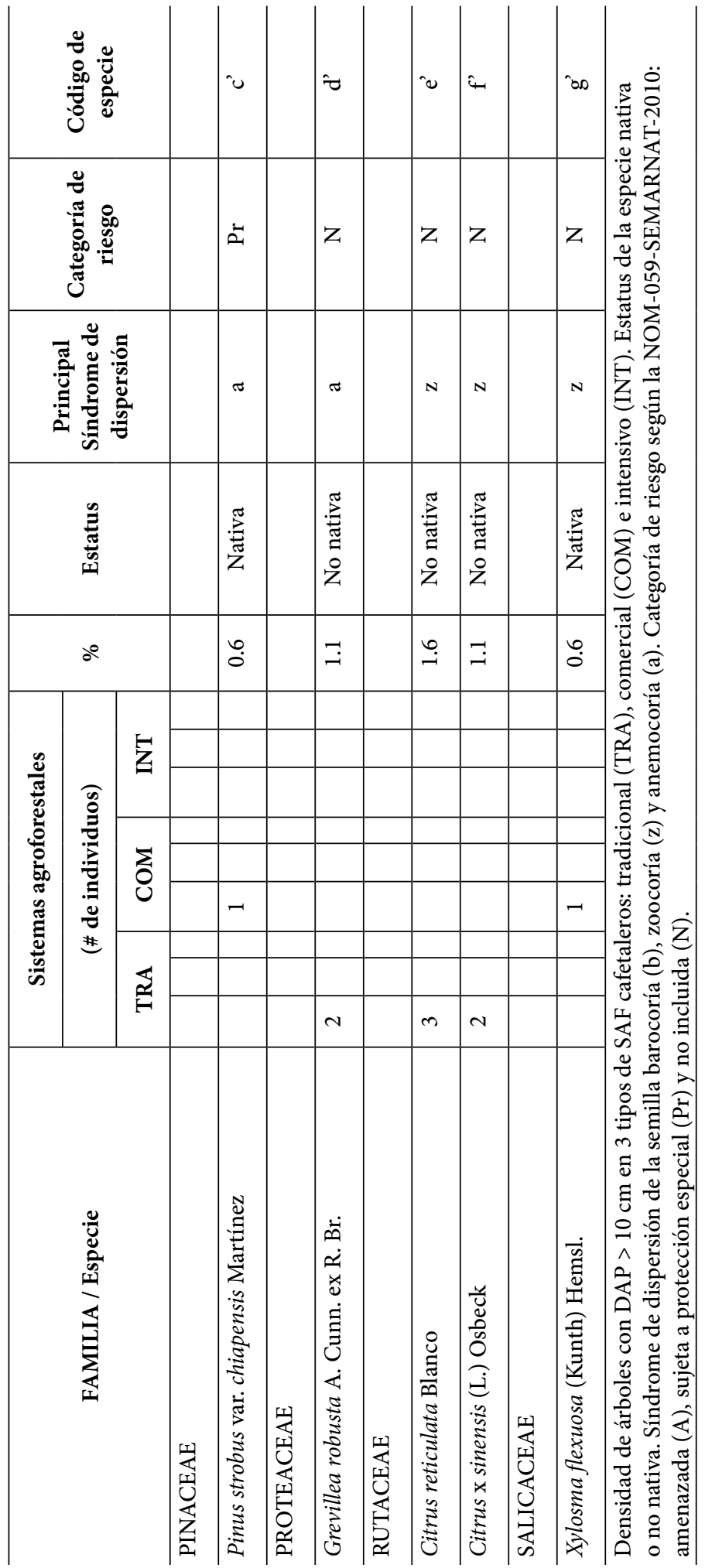


Las curvas de acumulación de especies de leñosas para la riqueza observada (Sobs) y los estimadores de riqueza (ACE y Jacknife 2) no alcanzaron la asíntota, solo el estimador de Chao1 tiende a dicho comportamiento (Figura 1). Las especies únicas - singletons- (11) y las especies duplicadas - doubletons - (7) representan el $55 \%$ de los árboles encontrados y tienden a incrementarse a medida que se acumula el esfuerzo de muestreo. Los estimadores de Chao 1, ACE y Jackknife 2 representaron el 79, 75 y 50 \% de las especies, respectivamente.

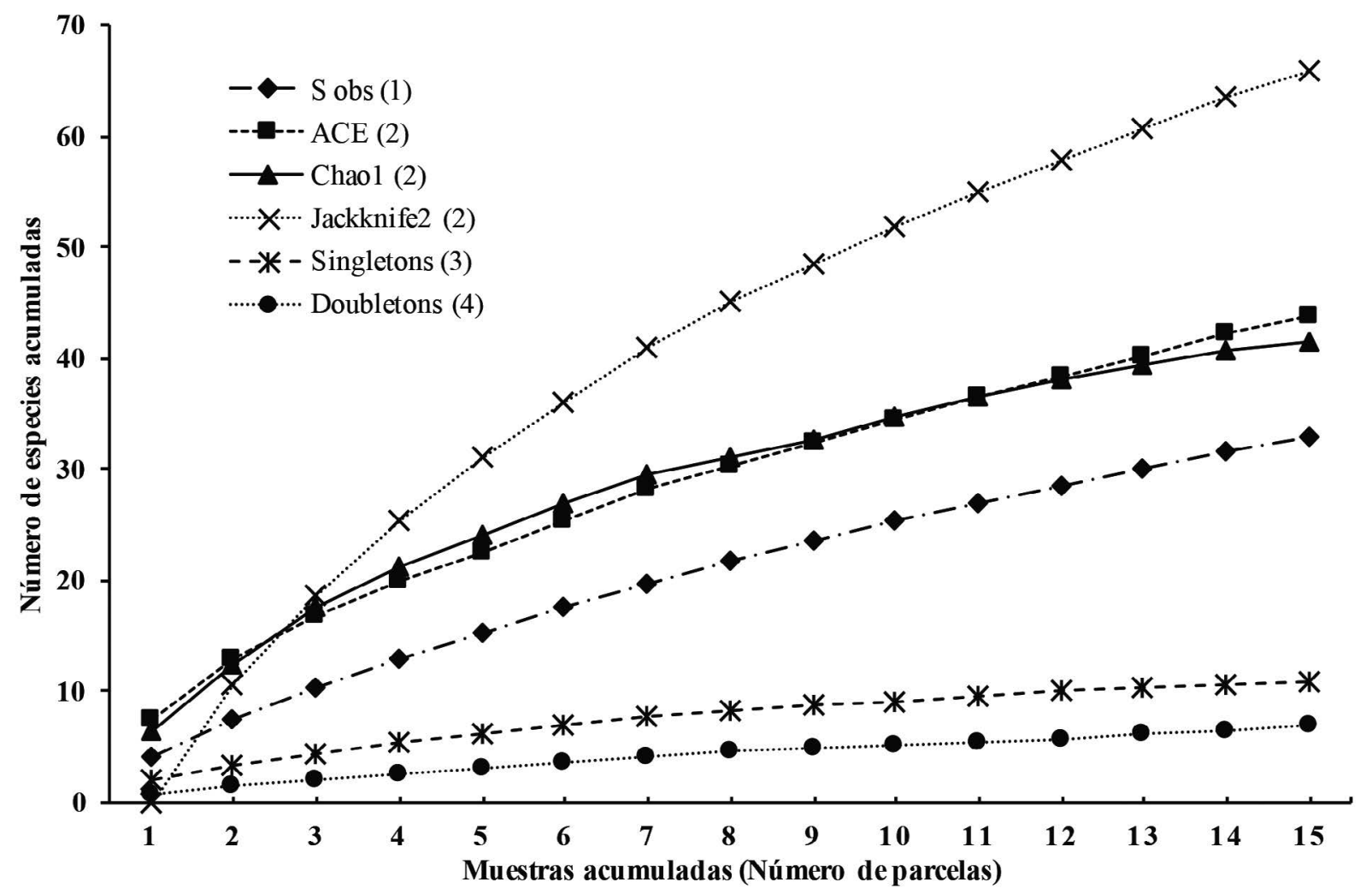

Figura 1. Curvas de acumulación de especies arbóreas ( $D A P \geq 10 \mathrm{~cm}$ ) para la totalidad de las parcelas de 0.1 ha en sistemas agroforestales cafetaleros con un gradiente de manejo tradicional, comercial e intensivo. (1) Número de especies observadas, (2) estimadores de riqueza no paramétricos, (3) especies únicas, (4) especies duplicadas

La abundancia total de individuos fue menor en el SAF tradicional (44 individuos) que en el comercial (62 individuos) y en el intensivo (72 individuos). Al comparar la riqueza efectiva de especies entre SAF, con referencia a la menor abundancia total de individuos (SAF tradicional), el número efectivo de especies $\left({ }^{0} \mathrm{D}\right)$ fue de 8.3 para el SAF intensivo, 11 para el tradicional y significativamente diferente con respecto al comercial $\left({ }^{0} \mathrm{D}=17.5\right)$ (línea punteada vertical, Figura 2a). La diversidad de especies medianamente abundantes, que corresponde al exponente de la diversidad de Shannon $\left({ }^{1} \mathrm{D}\right)$, fue de 8.3 para tradicional y 14 para comercial, difiriendo significativamente para el intensivo que presentó 3.6 (Figura 2b). De igual manera, los valores $\mathrm{de}^{2} \mathrm{D}$ (el inverso del índice de Simpson) muestran que el SAF intensivo presentó el menor número de especies dominantes $\left({ }^{2} \mathrm{D}=2.2\right)$, lo que difiere significativamente de los SAF tradicional 
$\left({ }^{2} \mathrm{D}=6.9\right)$ y comercial $\left({ }^{2} \mathrm{D}=10.3\right)$ (Figura $\left.2 \mathrm{c}\right)$. De acuerdo con los números de Hill de primer orden (sin ponderar la abundancia de las especies), se encontró que el SAF comercial es aproximadamente el doble más diverso que los SAF intensivo (11 especies menos) y tradicional (10 especies menos), encontrándose una diferencia significativa de la diversidad en comercial frente a intensivo y tradicional. Este comportamiento se acrecienta con la extrapolación de las curvas, al incrementar el esfuerzo de muestreo (Figura 2a). El número de familias promedio por parcela no presentó diferencias significativas entre tipos de manejo. No obstante, la densidad promedio de árboles por parcela difiere significativamente entre tipos de SAF (Cuadro 3).
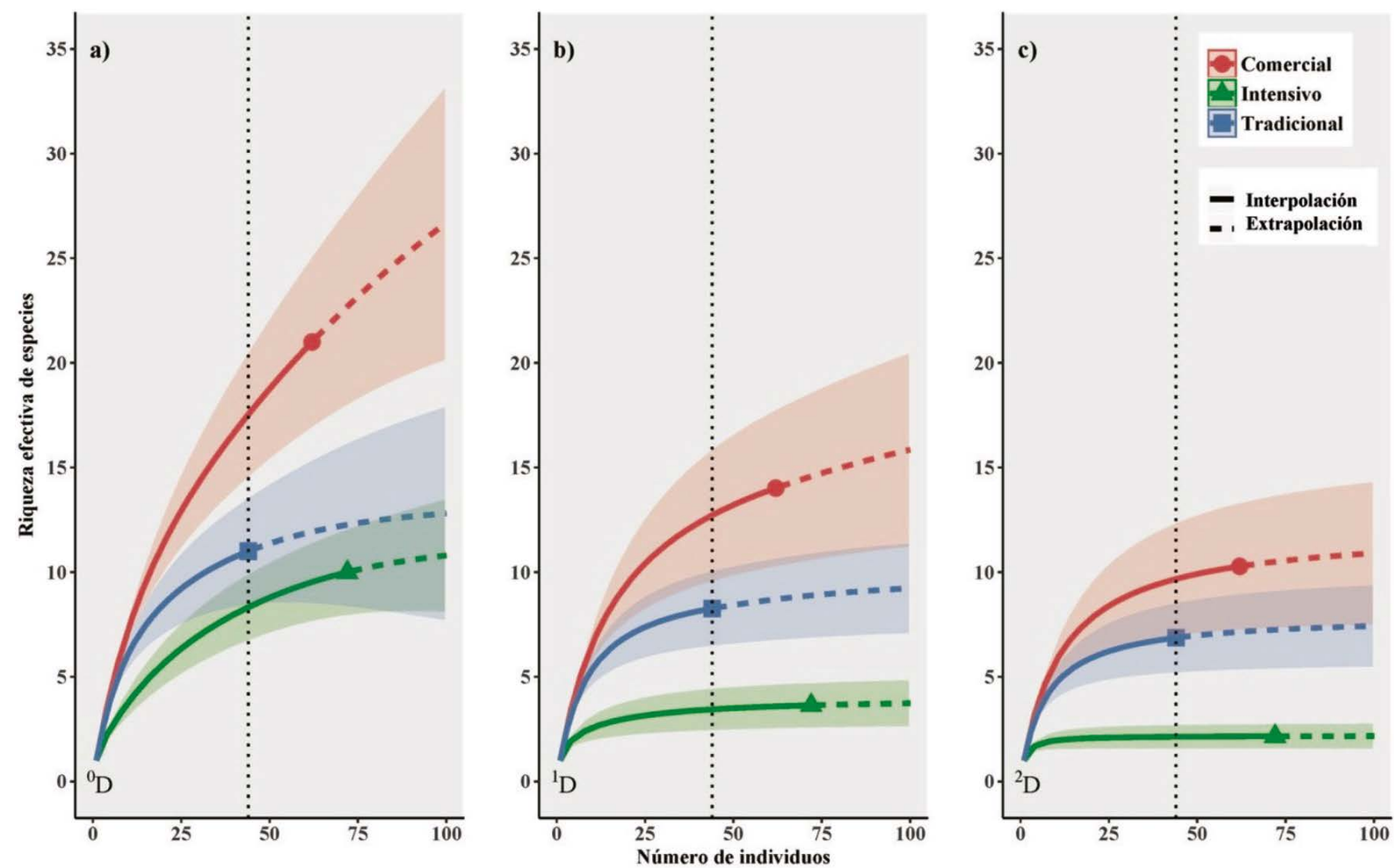

Figura 2. Curvas de interpolación (rarefacción)/extrapolación de la diversidad de árboles (DAP $\geq 10 \mathrm{~cm}$ ) en parcelas de 3 sistemas agroforestales (tradicional, comercial e intensivo), con 5 parcelas por tipo de sistema, con base en los números de Hill: a) ${ }^{0} \mathrm{D}$ : riqueza (número efectivo de especies), b) ${ }^{1} \mathrm{D}$ : exponente de la diversidad Shannon, c) ${ }^{2} \mathrm{D}$ : inverso del índice de diversidad de Simpson. La línea punteada vertical es de referencia al SAF tradicional que presentó la menor abundancia (44 individuos). Las bandas corresponden al intervalo de confianza. 
Cuadro 3. Análisis de varianza para las variables ecológicas en sistemas agroforestales de café con un gradiente de manejo en la Región Cafetalera de Coatepec, Veracruz, México

\begin{tabular}{l|l|l|l|l|l}
\hline & \multicolumn{5}{|c}{ Gradiente de manejo del SAF } \\
\hline Densidad de árboles por parcela & $\begin{array}{c}\text { Tradicional } \\
(\mathbf{n}=\mathbf{5})\end{array}$ & $\begin{array}{c}\text { Comercial } \\
(\mathbf{n}=\mathbf{5})\end{array}$ & $\begin{array}{c}\text { Intensivo } \\
(\mathbf{n}=\mathbf{5})\end{array}$ & F - estadística & \multicolumn{1}{|c}{ P - valor } \\
\hline Familias por parcela & $8.8( \pm 3.9) \mathrm{a}$ & $12.4( \pm 3.5) \mathrm{ab}$ & $14.4( \pm 1.5) \mathrm{b}$ & 4.05 & 0.045 \\
\hline Especies de árboles nativas & $2.2( \pm 0.8)$ & $3.8( \pm 1.8)$ & $3.2( \pm 0.8)$ & 2.13 & 0.162 \\
\hline Especies de árboles no nativos & $2.6( \pm 1.5)$ & $4.6( \pm 1.9)$ & $4.0( \pm 1.2)$ & 2.08 & 0.168 \\
\hline Individuos arbóreos nativos & $0.6( \pm 1.3)$ & $0.4( \pm 0.5)$ & $0.2( \pm 0.4)$ & 0.26 & 0.775 \\
\hline Individuos arbóreos no nativos & $7.4( \pm 4.6)$ & $9.3( \pm 5.5)$ & $14.2( \pm 1.3)$ & 3.29 & 0.073 \\
\hline Especies de árboles con síndrome zoocoría & $1.4( \pm 3.1)$ & $1.8( \pm 3.0)$ & $0.2( \pm 0.4)$ & 0.54 & 0.596 \\
\hline Especies de árboles con síndrome anemocoría & $0.4( \pm 0.5)$ & $1.4( \pm 1.1)$ & $0.6( \pm 0.5)$ & 2.21 & 0.152 \\
\hline Especies de árboles con síndrome barocoría & $0( \pm 0)$ & $0.2( \pm 0.4)$ & $0( \pm 0)$ & $\mathrm{sd}$ & $\mathrm{sd}$ \\
\hline Individuos arbóreos con síndrome zoocoría & $8.2( \pm 4.1) \mathrm{a}$ & $8.0( \pm 2.5) \mathrm{a}$ & $13( \pm 1.9) \mathrm{b}$ & 4.42 & 0.037 \\
\hline Individuos arbóreos con síndrome anemocoría & $0.6( \pm 0.9)$ & $3.4( \pm 3.4)$ & $1.4( \pm 1.5)$ & 2.09 & 0.166 \\
\hline Individuos arbóreos con síndrome barocoría & $0( \pm 0)$ & $1.0( \pm 2.2)$ & $0( \pm 0)$ & $\mathrm{sd}$ & $\mathrm{sd}$ \\
\hline Los promedios $( \pm$ d & $3.4( \pm 1.8)$ & $3.6( \pm 1.1)$ & 0.41 & 0.671 \\
\hline
\end{tabular}

Los promedios ( \pm desviación estándar) seguidos por distinta letra son significativamente diferentes entre tipologías (parcelas), por prueba de Tukey $(\mathrm{p}<0.05)$.

\subsection{Composición de las especies arbóreas}

El SAF comercial es el más rico (21 especies), en comparación con el tradicional (11 especies) y el intensivo (10 especies) (Figura 3). En el sistema comercial, se encuentra el mayor número de especies exclusivas (15) con el $46 \%$ del total de la riqueza. Las especies T. micrantha, I. vera y $H$. appendiculatus son compartidas entre las 3 tipologías (Cuadro 2). Los SAF más semejantes en cuanto a diversidad y abundancia arbórea (índice de Sørensen) son tradicional y comercial con solamente el $22 \%$ de similitud (5 especies compartidas) (Figura 3, Cuadro 4). La mayor complementariedad es entre intensivo y comercial con el 85 \% (Cuadro 4). Así mismo, el patrón de distribución de las abundancias (pendientes de las curvas) difiere entre los tipos de SAF cafetaleros, presentando un ajuste para los modelos Null en tradicional (AIC $=34,46$ ), Preemption en comercial (AIC $=62,46)$ y Zipf en intensivo (AIC =37,74) (Figura 4). Inga jinicuil fue la especie más abundante en tradicional (10 individuos) e Inga vera lo fue en comercial (13 individuos) e intensivo (48 individuos). El SAF comercial presentó el mayor número de especies más escasas (11) y el tradicional e intensivo tuvieron cada uno 3 diferentes especies escasas (Figura 4). La especie $H$. appendiculatus que se presenta como escasa en los SAF tradicional y comercial está en segundo orden de abundancia en intensivo; algo similar ocurre con T. micrantha que es escasa en tradicional, pero en comercial e intensivo está en el grupo de especies con abundancias intermedias. 


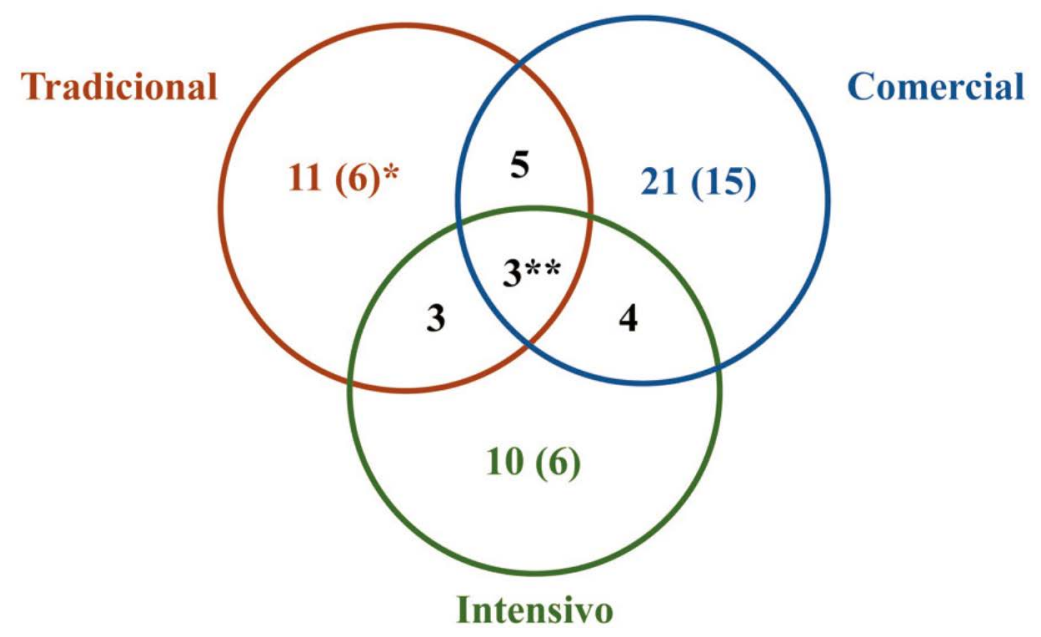

Figura 3. Diagrama de Venn para la distribución de las especies leñosas (DAP $\geq 10 \mathrm{~cm}$ ) en parcelas agroforestales de café con 3 tipos de manejo (tradicional, comercial e intensivo). ${ }^{\star}$ Especies por tratamiento, en paréntesis especies únicas. ${ }^{\star *}$ Especies compartidas entre tipos de manejo

Cuadro 4. Índices de similitud y complementariedad entre los tipos de manejo en sistemas agroforestales de café en el centro de Veracruz, México

\begin{tabular}{l|c|c|c}
\hline \multicolumn{1}{c|}{ SAF cafetalero } & Tradicional & Comercial & Intensivo \\
\hline Tradicional & - & $22 \%$ & $11 \%$ \\
\hline Comercial & $81 \%$ & - & $10 \%$ \\
\hline Intensivo & $83 \%$ & $85 \%$ & - \\
\hline
\end{tabular}

Los valores en la parte superior izquierda de la diagonal corresponden a la similitud (índice de Sørensen) expresada en porcentaje de semejanza. Los valores en cursiva en la parte inferior izquierda de la diagonal corresponden a la complementariedad (índice de complementariedad), variando de $0 \%$ cuando ambos sitios son idénticos a $100 \%$, al ser diferentes las especies en los 2 sitios.

No se registraron diferencias estadísticas entre la riqueza y abundancia de especies nativas y no nativas en los tipos de SAF (Cuadro 3). No obstante, el $90 \%$ de las especies arbóreas de los SAF comercial e intensivo fueron nativas y, en menor proporción, (73\%) ocurrieron en el tradicional. Se observaron cambios en el porcentaje de especies e individuos arbóreos con diferentes síndromes de dispersión de semillas. Sin embargo, el síndrome zoocoría dominó en los 3 tipos de SAF con valores entre el 46 y $90 \%$ para riqueza y abundancia, presentando diferencia significativa en la abundancia promedio por tipo de SAF, siendo mayor en SAF intensivo respecto a los SAF tradicional y comercial (Cuadro 3). El SAF tradicional presentó el porcentaje más alto (36\%) de especies e individuos leñosos con el síndrome anemocoría. El SAF intensivo no presentó especies con síndrome barocoría, pero, los SAF tradicional y comercial tuvieron valores entre el 10 y el $18 \%$ en el número de especies e individuos con este síndrome (del 46 al 60 \%). Juglans pyriformis se encuentra en categoría de riesgo amenazada; así mismo, Cupressus lusitanica, Cedrela odorata y Pinus strobus están sujetas a protección especial en la norma mexicana. Las restantes especies no se incluyen en la normatividad (Cuadro 2).

\begin{tabular}{|c|c|c|}
\hline 30 & $\begin{array}{l}\text { Creative Commons (Reconocimiento al autor-No comercial-Compartir igual } 4.0 \text { Internacional } \\
\text { (CC BY NC SA } 4.0 \text { Internacional) }\end{array}$ & $\begin{array}{l}\text { Revista de } \\
\text { CIENCIAS AMBIENTALES } \\
\text { Tropical Journal of Environmental Sciences }\end{array}$ \\
\hline
\end{tabular}




\section{Discusión}

\subsection{Riqueza, abundancia y diversidad}

La familia Fabaceae y, en especial, el género Inga en los 3 tipos de manejo estudiados presentan una abundancia alta, esto es característico de la mayoría de las zonas cafetaleras de México (López-Gómez et al., 2008; Bandeira, Martorell, Meave y Caballero, 2005), como consecuencia de la reducción de la estructura de los cafetales que buscan incrementar la producción (Perfecto y Vandermeer, 2015); en algunos casos, estas prácticas son promovidas por instituciones estatales. De igual forma, estas decisiones son fundamentadas y aceptadas por los productores y técnicos, debido a que las especies de este género tienen un efecto positivo en los SAF, al mejorar fertilidad en el suelo y favorecer las condiciones microclimáticas en los cafetos (Peeters et al., 2003; Romero-Alvarado et al., 2002; Roskoski, 1982; Siles et al., 2010). Sin embargo, es importante promover en las fincas establecidas en paisajes cafetaleros el uso de otras especies de árboles que no sean Inga spp., resaltando las ventajas para agricultores y sus plantaciones, al diversificar el componente leñoso en los SAF. Algunos beneficios son la recuperación de la fertilidad de los suelos, la regulación de plagas, la obtención de madera y alimentos y el incremento en el potencial de conservación de los SAF cafetaleros (Cerdán, Rebolledo, Soto, Rapidel y Sinclair, 2012).

También el tipo manejo de los SAF puede estar incidiendo en las especies y el número de individuos arbóreos (ensambles arbóreos, Figura 4) que el productor deja en sus predios, tanto es así que en este estudio la densidad de árboles por parcela y el número de individuos de las especies más abundantes de cada SAF tienden a incrementarse en los SAF con menor densidad de cafetos, plantas más viejas, menos podadas y con más variedades utilizadas (Cuadro 3, Figura 4). Al hablar de producción bajo sombra, es importante diferenciar entre diversidad arbórea, densidad arbórea y porcentaje de sombra. Esto se debe a que una mayor densidad de árboles con copas densas, pero adecuadamente podadas, tendrá los mismos rendimientos de café que un cafetal que presente una escasa densidad de árboles de sombra sin podas (Soto-Pinto, Perfecto, Castillo-Hernández y Caballero-Nieto, 2000). 
Revista de Ciencias Ambientales (Trop J Environ Sci). (Julio-Diciemnbre, 2018). EISSN: 2215-3896. Vol 52(2): 16-38.

DOI: http://dx.doi.org/10.15359/rca.52-2.2

URL: www.revistas.una.ac.cr/ambientales

EMAIL: revista.ambientales@una.cr
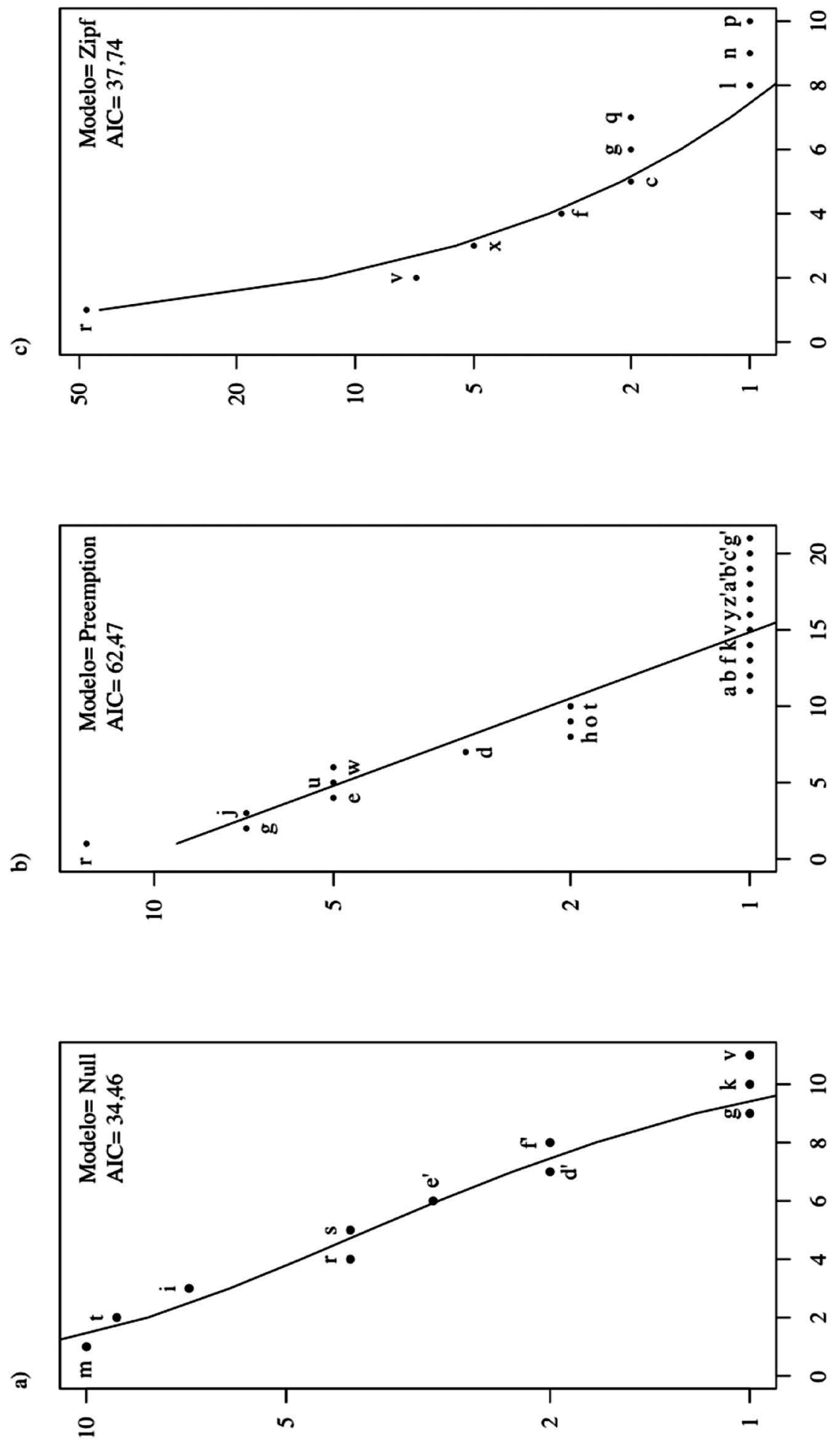

ẹpurpunqv

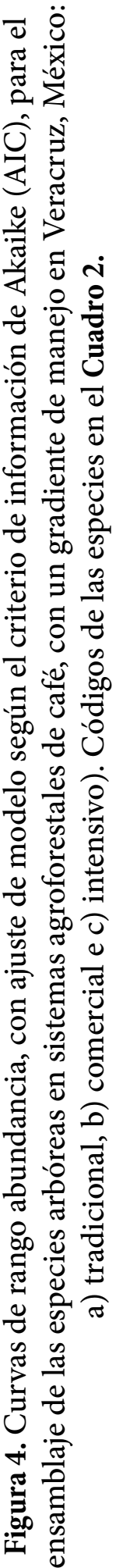


De las especies arbóreas encontradas en los SAF estudiados, Roseodendron donnell-smithii, Cordia alliodora, Cupressus lusitanica, Inga paterno, Ficus aurea, F. crocata y Pinus strobus var. Chiapensis no han sido reportadas como especies de sombrío para el café en la Región de Coatepec (López-Gómez et al., 2008). Esto se podría deber a que algunas especies no ocurren de forma natural para la zona y han sido introducidas por tener un valor económico, lo que principalmente se observó en los SAF con manejo comercial. En otras regiones cafetaleras del estado de Veracruz, C. alliodora es una de las especies más importantes en la estructura de los SAF de café, por el valor económico que tiene la madera (García-Mayoral et al., 2015). En esta misma región, el Instituto Nacional de Investigaciones Forestales, Agrícolas y Pecuarias realiza ensayos para evaluar el desempeño de especies maderables como sombrío de los cafetos, las cuales, posteriormente, serán impulsadas entre productores, lo que incidiría en el ensamblaje arbóreo presente en los SAF (López, Salazar, Martínez, Padilla y García, 2013b), como lo ocurrido a escala del paisaje y en la ecología del café con la difusión de algunas estrategias de manejo de sombra por el desaparecido Instituto Mexicano del Café de México (INMECAFE).

La abundancia de los árboles, que es significativamente diferente entre los SAF intensivos y los tradicionales, está determinada fuertemente por el tipo de las especies (Figura 4), puede haber alta abundancia con individuos pequeños de copas ralas (y podadas para Inga) y baja abundancia para especies altas que no pueden ser podadas fácilmente, lo que coincide con López-Gómez et al. (2008) y Méndez et al. (2007). Inga vera, siendo la especie dominante en el manejo intensivo, podría incidir en este resultado, ya que su arquitectura es de porte bajo a medio, copa aplanada, amplia, muy extendida y con follaje ralo (Standley y Stermarck, 1946), lo que facilita su manejo en comparación con otras especies, un criterio de selección por los productores (Cerdán et al., 2012). En los SAF comerciales e intensivos, se realizan podas a los árboles, lo que les permite manejar un mayor número de estos en las fincas (Cuadro 3). En un estudio para la zona, se menciona que los productores del sistema de monocultivo sombreado realizan podas intensivas, lo que condiciona estos parámetros en las plantaciones de café (López-Gómez et al., 2008). Adicionalmente, los productores que tienen un manejo comercial integran en los SAF especies maderables como una inversión a futuro; esta práctica se registra en plantaciones del sur de México, donde los cafeticultores tradicionales promueven a Cedrela odorata por su valor comercial y calidad bien conocida de la madera (Peeters et al., 2003). Los productores con manejo intensivo se centran en el café como principal actividad comercial, por lo que, en el criterio de elección de los árboles, predominan la facilidad de manejo, beneficios en fertilidad por medio de los aportes de hojarasca y las relaciones positivas con los microorganismos del suelo, características que también son expresadas por productores del sur de México (Soto-Pinto et al., 2007), de El Salvador (Méndez et al., 2007) y de Costa Rica (Cerdán et al., 2012).

\subsection{Composición de las especies arbóreas}

Los 3 tipos de SAF cafetaleros mostraron una ocurrencia de especies nativas del 73 al $90 \%$, siendo los SAF tradicionales los de menos especies nativas. Esto se debe a que los productores también utilizan como sombrío del café especies no nativas de valor comercial (Grevillea robusta) o para autoconsumo (Citrus reticulata y C. $\mathrm{x}$ sinensis). Esta situación ya ha sido reportada

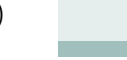


en otros trabajos en México (Bandeira et al., 2005; López-Gómez et al., 2008), lo que evidencia que la diversidad arbórea presente en los SAF estudiados contribuye a la provisión de servicios ecosistémicos y es reservorio de especies leñosas nativas e incluso algunas con prioridad para su conservación (Cuadro 2). En los SAF estudiados, los valores reportados en el número de especies e individuos con el síndrome zoocoría representa que estos agroecosistemas pueden brindar fuentes alimenticias para aves, mamíferos e insectos. Varios estudios evidencian el importante papel de los cafetales diversificados en la conservación de la fauna (Bali, Kumar y Krishnaswamy, 2007; Philpott y Bichier, 2012). Sin embargo, el número de individuos con síndrome zoocoría fue mayor a lo reportado por López-Gómez et al. (2008), quienes encontraron predominantemente el síndrome barocoría.

Todos los tipos de SAF se ajustaron a diferentes modelos de distribución con respecto a las abundancias observadas. El SAF tradicional presentó un ajuste al modelo Null, que es la expresión biológica de una distribución al azar relativamente equitativa de las especies en la comunidad (MacArthur, 1957). Se ajusta muy bien a poblaciones en equilibrio, de organismos con tamaño relativamente grande, larga vida y fuertemente competidores (Jaksic y Marone, 2007). Los árboles presentes en el sistema tradicional son de tallo más grueso y presentan mayor altura en comparación con individuos de los otros SAF estudiados. Además, son los SAF menos perturbados porque tienen menor manejo agronómico y también hay un número mayor de cafetos con edades avanzadas. El modelo Preemption presente en el SAF comercial denota que ocurren unas pocas especies arbóreas dominantes y las restantes, que son la gran mayoría, prácticamente son raras, lo que origina una distribución de recursos menos equitativa comparada con los otros modelos (Whittaker, 1965). Lo anterior podría indicar un patrón básicamente en estadios tempranos de una sucesión o en ambientes desfavorables (Jaksic y Marone, 2007). El ajuste del modelo Zipf en el SAF intensivo podría ser el resultado de que las parcelas muestreadas están en constante disturbio por el manejo agronómico que tienen los SAF (altas densidades de cafetos, control de arvenses, así como podas a los cafetos y árboles), es por ello que las comunidades vegetales se encuentran en un continuo proceso de sucesión. Este modelo ha tenido éxito en ajustarse a datos de comunidades durante un periodo de sucesión en el que las especies tardías ocurren en gran número, pero, al tener requerimientos complejos, resultan con abundancias muy bajas.

\section{Conclusiones}

El ensamble de las especies arbóreas en los SAF cafetaleros es influenciado por las decisiones que toman los productores, quienes principalmente dirigen sus esfuerzos a la productividad de los cafetos. Los SAF manejados intensivamente presentan una mayor abundancia de individuos arbóreos, mientras que los manejados tradicionalmente muestran una mayor diversidad y riqueza de especies. Se recomienda que se integren en las clasificaciones de los sistemas agroforestales tanto variables relacionadas con el componente arbóreo como al arbustivo. Esto permitirá clasificar, de manera sencilla, el estado actual de los SAF cafetaleros, para identificar aspectos (ecológicos y de manejo) relevantes por ser implementados en las zonas productoras. 


\section{Agradecimientos}

Damos las gracias a los dueños de las fincas de café incluidas en este estudio, por su autorización para trabajar en sus plantaciones. Agradecemos a los ingenieros Luis Monfil y Aldo Gómez-Moreno, por su apoyo en la colecta de datos para el desarrollo de este estudio. Al Dr. Miguel de Jesús Cházaro-Basáñez (Universidad Veracruzana) y al maestro Carlos Duran (Instituto de Ecología), quienes nos ayudaron a identificar los especímenes vegetales. Al Consejo de Ciencia y Tecnología de México (CONACYT) y a la Universidad del Tolima (Colombia), por el apoyo durante los estudios de doctorado de Mario J. Gómez Martínez. Además, a las personas revisoras anónimas de la revista, por sus aportes, los cuales enriquecieron el presente documento.

\section{Referencias}

Altieri, M. A., Nicholls, C. I., Henao, A. y Lana, M. A. (2015). Agroecology and the design of climate change-resilient farming systems. Agronomy for Sustainable Development, 35(3), 869890. Doi: $10.1007 / \mathrm{s} 13593-015-0285-2$

Angiosperm Phylogeny Group IV [APG IV]. (2016). An update of the Angiosperm Phylogeny Group classification for the orders and families of flowering plants: APG IV. Botanical Journal of the Linnean Society, 181(1), 1-20. Doi: 10.1111/boj.12385

Avelino, J., Cristancho, M., Georgiou, S., Imbach, P., Aguilar, L., Bornemann, G.... Morales, C. (2015). The coffee rust crises in Colombia and Central America (2008-2013): impacts, plausible causes and proposed solutions. Food Security, 7(2), 303-321. Doi: 10.1007/ s12571-015-0446-9

Bali, A., Kumar, A. y Krishnaswamy, J. (2007). The mammalian communities in coffee plantations around a protected area in the Western Ghats, India. Biological Conservation, 139(1-2), 93-102. Doi: 10.1016/j.biocon.2007.06.017

Bandeira, F. P., Martorell, C., Meave, J. A. y Caballero, J. (2005). The role of rustic coffee plantations in the conservation of wild tree diversity in the Chinantec region of Mexico. Biodiversity and Conservation, 14(5), 1225-1240. Doi: 10.1007/s10531-004-7843-2

Chao, A., Gotelli, N. J., Hsieh, T. C., Sander, E. L., Ma, K. H., Colwell, R. K. y Ellison, A. M. (2014). Rarefaction and extrapolation with Hill numbers: a framework for sampling and estimation in species diversity studies. Ecological Monographs, 84(1), 45-67. Doi: 10.1890/130133.1, 45-67

Charbonnier, F., Roupsard, O., le Maire, G., Guillemot, J., Casanoves, F., Lacointe, A., ... Dreyer, E. (2017). Increased Light-Use Efficiency Sustains Net Primary Productivity of Shaded Coffee Plants in Agroforestry System. Plant, Cell \& Environment, 40(8), 1592-1608. Doi: 10.1111/pce.12964

Cerdán, C. R., Rebolledo, M. C., Soto, G., Rapidel B. y Sinclair, F. L. (2012). Local knowledge of impacts of tree cover on ecosystem services in smallholder coffee production systems. Agricultural Systems, 110, 119-130. Doi: 10.1016/j.agsy.2012.03.014 
Revista de Ciencias Ambientales (Trop J Environ Sci). (Julio-Diciemnbre, 2018). EISSN: 2215-3896. Vol 52(2): 16-38.

DOI: http://dx.doi.org/10.15359/rca.52-2.2

URL: www.revistas.una.ac.cr/ambientales

EMAIL: revista.ambientales@una.cr

Colwell, R. K. (2013). Estimates: statistical estimation of species richness and shared species from samples. Version 9. Colorado, USA: University of Colorado Boulder. Recuperado de http:// purl.oclc.org/estimates

Colwell, R. K. y Coddingtong, J. A. (1994). Estimating terrestrial biodiversity through extrapolation. Philosophical transactions of the Royal Society of London, 345(1311), 101-108. Doi: 10.1098/rstb.1994.0091

Escamilla, P. E., Licona, A. L., Díaz, S., Santoyo, V. H., Sosa, R. y Rodríguez, R. (1995). Los sistemas de producción de café en el centro de Veracruz, México. Un análisis tecnológico. Revista de Historia, 30, 41-67.

García-Mayoral, L. E., Valdez-Hernández, J. I., Luna-Cavazos, M. y López-Morgado, R. (2015). Estructura y diversidad arbórea en sistemas agroforestales de café en la sierra de Atoyac, Veracruz. Madera y Bosques, 21(3), 69-82.

Gordon, C., Manson, R., Sundberg, J. y Cruz-Angón, A. (2007). Biodiversity, profitability, and vegetation structure in a Mexican coffee agroecosystem. Agriculture, Ecosystems \& Environment, 118(1), 256-266. Doi: 10.1016/j.agee.2006.05.023

Hill, M. O. (1973). Diversity and evenness: a unifying notation and its consequences. Ecology, 54(2), 427-432. Doi: 10.2307/1934352

Hsieh, T. C., Ma, K. H. y Chao, A. (2016). iNEXT: an R package for rarefaction and extrapolation of species diversity (Hill numbers). Methods in Ecology and Evolution, 7(12), 1451-1456. Doi: 10.1111/2041-210X.12613

Jaksic, F. y Marone, L. (2007). Ecología de comunidades. Santiago, Chile: Ediciones Universidad Católica de Chile.

Jost,L.(2006).Entropyanddiversity.Oikos, 113(2),363-375.Doi:10.1111/j.2006.0030-1299.14714.x

Jost, L., Chao, A. y Chazdon, R. L. (2011). Compositional similarity and $\beta$ (beta) diversity. En A. Magurran y B. J. McGill (Eds.), Biological diversity: frontiers in measurement and assessment (pp. 66-87). New York, USA: Oxford University Press.

López, R., Escamilla, E., Díaz, G., Guajardo, R., Martínez, J., García, L., ... Barreda, S. (2013a). La cafeticultura en México y su problemática. En R. López, G. Díaz y A. Zamarripa (Eds.), El sistema productor de café en México: problemática y tecnología de reproducción (pp. 5-31). CDMX, México: SAGARPA-INIFAP.

López, R., Salazar, J. G., Martínez, J. L., Padilla, G. y García, L. E. (2013b). Producción de café bajo sistemas agroforestales (SAF-café): caso de la zona centro del estado de Veracruz. En R. López, G. Díaz y A. Zamarripa (Eds.), El sistema productor de café en México: problemática y tecnología de reproducción (pp. 315-346). CDMX, México: SAGARPA-INIFAP. 
López-Gómez, A. M. y Williams-Linera, G. (2006). Evaluación de métodos no paramétricos para la estimación de riqueza de especies de plantas leñosas en cafetales. Boletín de la Sociedad Botánica de México, 78, 7-15.

López-Gómez, A. M., Williams-Linera, G. y Manson, R. H. (2008) Tree species diversity and vegetation structure in shade coffee farms in Veracruz, Mexico. Agriculture, Ecosystems \& Environment, 124(3), 160-172. Doi: 10.1016/j.agee.2007.09.008

MacArthur, R. H. (1957). On the relative abundance of bird species. Proceedings of the National Academy of Sciences of the United States of America, 43, 293-295.

Magurran, A. E y McGill, B. J. (2011). Biological diversity: frontiers in measurement and assessment. Oxford, United Kingdom: Oxford University Press.

Matson, P. A., Parton, W. J., Power, A. G. y Swift, M. J. (1997). Agricultural intensification and ecosystem properties. Science, 277(5325), 504-509. Doi: 10.1126/science.277.5325.504

Marin-Castro, B. E., Geissert, D., Negrete-Yankelevich, S. y Chávez, A. G. T. (2016). Spatial distribution of hydraulic conductivity in soils of secondary tropical montane cloud forests and shade coffee agroecosystems. Geoderma, 283, 57-67. Doi: 10.1016/j.geoderma.2016.08.002

Méndez, V. E., Gliessman, S. R. y Gilbert, G. S. (2007). Tree biodiversity in farmer cooperatives of a shade coffee landscape in western El Salvador. Agriculture, Ecosystems \& Environment, 119(1), 145-159. Doi: 10.1016/j.agee.2006.07.004

Oksanen, F. J., Blanchet, G., Kindt, R., Legendre, P., Minchin, R. P., O’Hara, R. B., ... Wagner, H. (2017). Package VEGAN, version 2.4-3. Recuperado de http://cran.r-project.org/web/packages/vegan/index.html

Peeters, L. Y. K., Soto-Pinto, L., Perales, H., Montoya, G. y Ishiki, M. (2003). Coffee production, timber, and firewood in traditional and Inga-shaded plantations in Southern Mexico. Agriculture, Ecosystems \& Environment, 95(2), 481-493. Doi: 10.1016/S0167-8809(02)00204-9

Pennington, T. y Sarukhán, J. (2005). Árboles tropicales de México: manual para la identificación de las principales especies. CDMX, México: Fondo de Cultura Económica.

Perfecto, I. y Vandermeer, J. (2015). Coffee agroecology: A new approach to understanding agricultural biodiversity, Ecosystem Services and Sustainable Development. New York, USA: Routledge.

Philpott, S. M., Arendt, W. J., Armbrecht, I., Bichier, P., Diestch, T. V., Gordon, C., Zolotoff, J. M. (2008). Biodiversity loss in Latin American coffee landscapes: Review of the evidence on ants, birds, and trees. Conservation Biology, 22(5), 1093-1105. Doi: 10.1111/j.1523-1739.2008.01029.x

Philpott, S. M. y Bichier, P. (2012). Effects of shade tree removal on birds in coffee agroecosystems in Chiapas, Mexico. Agriculture, ecosystems \& environment, 149, 171-180. Doi: 10.1016/j. agee.2011.02.015 
Revista de Ciencias Ambientales (Trop J Environ Sci). (Julio-Diciemnbre, 2018). EISSN: 2215-3896. Vol 52(2): 16-38.

DOI: http://dx.doi.org/10.15359/rca.52-2.2

URL: www.revistas.una.ac.cr/ambientales

EMAIL: revista.ambientales@una.cr

R Core Team. (2017). R: A language and environment for statistical computing. R. Vienna, Austria: Foundation for Statistical Computing. Recuperado de https://www.R-project.org/

Romero-Alvarado, Y., Soto-Pinto, L., García-Barrios, L. y Barrera-Gaitán, J. F. (2002). Coffee yields and soil nutrients under the shades of Inga sp. vs. multiple species in Chiapas, Mexico. Agroforestry Systems, 54(3), 215-224. Doi: 10.1023/A:101601373015

Roskoski, J. P. (1982). Nitrogen fixation in a Mexican coffee plantation. Plant and Soil, 67, 283291. Doi: $10.1007 / B F 02182775$

Siles, P., Harmand, J. M. y Vaast, P. (2010). Effects of Inga densiflora on the microclimate of coffee (Coffea arabica L.) and overall biomass under optimal growing conditions in Costa Rica. Agroforestry Systems, 78(3), 269-286. Doi: 10.1007/s10457-009-9241-y

Soto-Pinto, L., Perfecto, I., Castillo-Hernández, J. y Caballero-Nieto, J. (2000). Shade effect on coffee production at the northern Tzeltal zone of the state of Chiapas, Mexico. Agriculture, Ecosystems \& Environment, 80(1), 61-69. Doi:10.1016/S0167-8809(00)00134-1

Soto-Pinto, L., Villalvazo-López, V., Jiménez-Ferrer, G., Ramírez-Marcial, N., Montoya, G., y Sinclair, F. (2007). The role of local knowledge in determining shade composition of multiestrata coffee systems in Chiapas. Biodiversity and Conservation, 16(2), 419-436. Doi:10.1007/ s10531-005-5436-3

Sousa, S. (2009). Adiciones al género Inga (Ingeae, Mimosoideae, Leguminosae) para la flora mesoamericana. Acta Botánica Mexicana, 89, 25-41.

Standley, P. y Stermarck, J. (1946). Flora of Guatemala, Fieldiana: Botany, Volume 24, Part V. Chicago, USA: Chicago Natural History Museum.

Tropicos.org. (2017). Missouri Botanical Garden. Missouri, USA. Recuperado de http://www. tropicos.org

Tscharntke, T., Clough, Y., Bhagwat, S. A., Buchori, D., Faust, H., Hertel, D., ... Wanger, T. C. (2011). Multifunctional shade-tree management in tropical agroforestry landscapes - a review. Journal of Applied Ecology, 48(3), 619-629. Doi: 10.1111/j.1365-2664.2010.01939.x

Whittaker, R. H. (1965). Dominance and diversity in land plant communities. Science, 147(3655), 250-260. Doi: 10.1126/science.147.3655.250 